\title{
Earth Mover's Morphing: Topology-Free Shape Morphing Using Cluster-Based EMD Flows
}

\author{
Yasushi Makihara and Yasushi Yagi \\ Osaka University, 8-1 Mihogaoka, Ibaraki, Osaka, 567-0047, Japan
}

\begin{abstract}
This paper describes a method for topology-free shape morphing based on region cluster-based Earth Mover's Distance (EMD) flows, since existing methods for closed curve/surface-based shape morphing are inapplicable to regions with different genera. First, the shape region is decomposed into a number of small clusters by Fuzzy C-Means clustering. Next, the EMD between the clusters of two key shapes is calculated and the resultant EMD flows are exploited as a weighted manyto-many correspondence among the clusters. Then, the fuzzy clusters are transported based on the EMD flows and a transition control parameter. Unlike the closed curve/surface-based methods, the morphs using cluster transportation are not guaranteed to be a binary image, and hence graph cut-based binary denoising is applied to a volumetric image of the two-dimensional position and the one-dimensional transition control parameter. The experiments demonstrate that the proposed method can perform morphing between shapes with different genera, such as walking silhouettes or alphabetical characters.
\end{abstract}

\section{Introduction}

For a long time, image morphing [1] has attracted much attention in the image processing and computer graphics fields, because it serves as a powerful image/video editing tool for creating unique visual effects in view morphing [2] and 3D face synthesis [3]. Image morphing techniques are further used in computer vision and pattern recognition areas to generate view-interpolated images for efficient supervised learning [4] and training samples for deformable shape matching [5] [6].

In the early stages of morphing research, correspondences between geometric primitives including points, lines, and curves were manually given and various types of warping functions were proposed, such as mesh warping [7], field morphing [8], the radial basis function [9], thin plate spline [10], energy minimizationbased function [11], and Multilevel Free-Form Deformation (MFFD) [12] [13]. During the middle stages, methods for automatic correspondence were proposed to reduce burdens of user input [14] [15] [16] [17] [18]. As the above methods rely on image texture, they are not applicable to shape morphing without texture.

On the other hand, the shape morphing problem is treated predominantly as a shape contour/surface deformation problem because of its compact expression [19] [20]. The morphing target is, however, limited to shapes with the same 


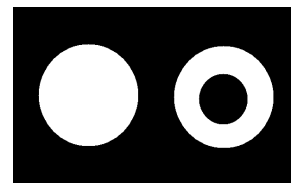

(a) Disk and annulus

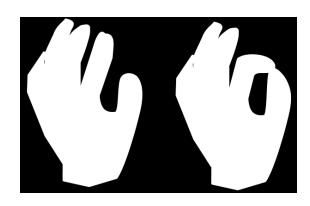

(b) Hand

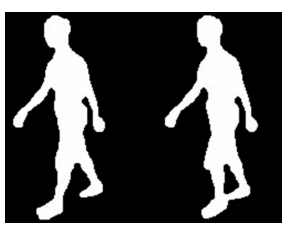

(c) Gait

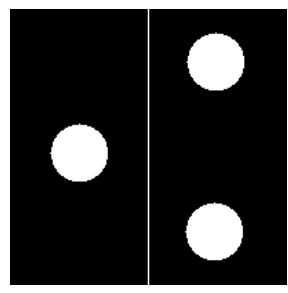

(d) Split disks

Fig. 1: Examples of pairs of shapes: (a), (b) and (c) with different genera (left: 0 genera, right: 1 genus), and (d) with a different number of shapes.

genus; in other words, most of the existing methods cannot deal with morphing of shapes with different genera, such as morphing from a disk to an annulus in a 2D domain (Fig. 1(a)) or from a ball to a torus in a 3D domain. Indeed, this limitation is critical for many silhouette-based applications including hand posture recognition (Fig. 1(b)), gait recognition (Fig. 1(c)), and action recognition. Although several methods [21] [22] can treat such topology differences, they suffer still from a tedious process which requires significant user input.

To cope with such topological changes, volume-based approaches were proposed and they generally fall into two categories, distance field approaches [23] [24] and level-set approaches [25] [26].

The distance field approaches first constructs a signed distance field to contour/surface for each shape and then generates an intermediate contour/surface by interpolating the signed distance fields. It is, however, reported that the distance field approaches sometimes produce undesirable pop-up artifacts [25]. For example, in case of morphing from a single disk to two distantly split disks (Fig. $1(\mathrm{~d})$ ), while the center disk disappears by erosion, the two split disks emerge as points and are dilated to the destination disks.

The level-set approaches also constructs the signed distance field and then a contour/surface is evolved based on a partial differential equation within socalled "narrow band" in the Level-Set Method (LSM) [27]. Because the narrow band gradually moves in the evolution process and it never pops up from the other region, it sometimes fails in reaching the destination shape. For example, in case of morphing from a single disk to two distantly split disks (Fig. 1(d)), the two split disks never emerge because the narrow band disappears after the center disk erosion and does not pop up from a region of the two split disk.

Consequently, we propose a method for topology-free shape morphing based on region cluster-based EMD flows in a 2D domain. The shape region is first decomposed into a set of small clusters and then EMD flows between the clusters of two key shapes are calculated. Each cluster is morphed and blended according to the many-to-many correspondence of the EMD flows and transition control parameter. Since the proposed method relies on the EMD flows, that is, a kind of warping motion, it is applicable not only to shapes with different topologies, 
but also to a different number of shapes as in (Fig. 1(d)), which results in more interesting split-process morphing as shown in Fig. 10.

\section{Related work}

Automatic morphing of textured image: Gao et al. [14] proposed an energy minimization approach based on image feature consistency and deformation amount, while Tekalp [16] and Toshev et al. [15] exploited optical flow-based feature correspondences and saliency region correspondences, respectively. Chen et al. [17] exploited pixel correspondence based on range data and camera pose for view interpolation. Shechtman et al. [18] proposed a regenerative morphing from small pieces of the two source images based on source similarity and time coherence. Zhu et al. [28] [29] formulated morphing of textured images as optimal mass transportation problem and solved it in iterative energy minimization framework.

Polygonal/polyhedral shape morphing: Sederberg [19] proposed a 2D polygonal shape morphing method based on work minimization of the vertex deformation. Kent et al. [20] extended the idea to 3D polyhedral shape and computed a transformation process by interpolating between corresponding vertex positions. These methods is, however, not applicable to free-form shape.

Non-rigid shape matching and registration In addition to pure morphing techniques, it is possible to include shape contour matching techniques, such as those based on geodesic distance [30] or the Earth Mover's Distance (EMD) [31], in the shape morphing, as the matching results give the correspondence of each point on the contour. Non-rigid shape registration is also related to contour/surface-based shape morphing. Non-rigid shape is usually expressed as line segments for a $2 \mathrm{D}$ shape or surface meshes for a $3 \mathrm{D}$ shape and the correspondences of the contour/surface between two shapes are obtained in the registration process based on minimum distortion criteria [32], a data-driven deformation prior [33], or a elastic convolved ICP [34]. Then, interpolated nonrigid shapes can be generated based on the correspondences. These methods are, however, not applicable to shapes with different genera.

\section{Earth Mover's Morphing}

\subsection{Construction of floating-bin histogram}

The first step involves constructing a floating-bin histogram from a shape silhouette. First, the 2D position in the image is defined as $\boldsymbol{x}=[x, y]^{T}$ and subsequently, a silhouette image $I(\boldsymbol{x})$ is defined as

$$
I(\boldsymbol{x})= \begin{cases}1 & \text { for inside shape } \\ 0 & \text { for outside shape }\end{cases}
$$

The shape silhouette can also be expressed as a set of points within the shape as $X_{s}=\{\boldsymbol{x} \mid I(\boldsymbol{x})=1\}$. In addition, an area $A(I)=\sum_{\boldsymbol{x}} I(x)$ and area-normalized weight $w(\boldsymbol{x})=I(\boldsymbol{x}) / A(I)$ are calculated in preparation of our formulation. 

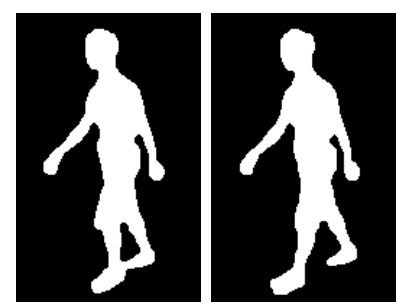

(a) Key shapes

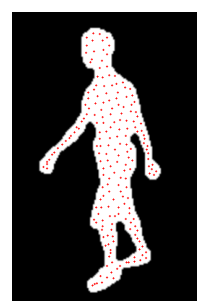

(b) Cluster means
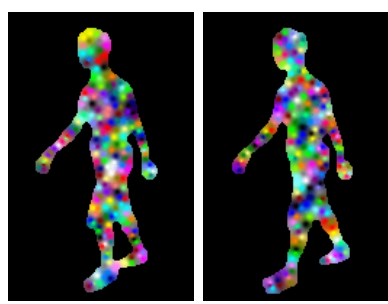

(c) Membership

Fig. 2: Results of Fuzzy C-Means clustering. In each pair, the left and right images are the source and destination, respectively. Cluster mean positions are depicted as a red cross in (b). In (c), each color corresponds to a cluster and the membership for all the clusters is depicted with alpha blending.

Next, $N_{c}$ fuzzy clusters are obtained by Fuzzy C-Means (FCM) clustering [35]. The reason that fuzzy clusters are chosen instead of a hard clustering method such as k-means clustering, is the effectiveness of the fuzzy property in the denoising process in the final step described in 3.4.

Let the $c$ th clusterfs mean, weight, and membership at $\boldsymbol{x}$ be $\overline{\boldsymbol{x}}_{c}, \bar{w}_{c}$, and $m_{c}(\boldsymbol{x})$, respectively, which satisfy

$$
\begin{aligned}
\overline{\boldsymbol{x}}_{c} & =\frac{\sum_{\boldsymbol{x} \in X_{s}} m_{c}(\boldsymbol{x}) \boldsymbol{x}}{\sum_{\boldsymbol{x} \in X_{s}} m_{c}(\boldsymbol{x})} \\
w_{c} & =\frac{\sum_{\boldsymbol{x} \in X_{s}} m_{c}(\boldsymbol{x}) w(\boldsymbol{x})}{\sum_{\boldsymbol{x} \in X_{s}} m_{c}(\boldsymbol{x})} \\
\sum_{c=1}^{N_{c}} m_{c}(\boldsymbol{x}) & =1 .
\end{aligned}
$$

Thus, a floating-bin histogram is composed of a set of bin means $\overline{\boldsymbol{X}}=\left\{\overline{\boldsymbol{x}}_{c}\right\}$ and a set of bin weights $\boldsymbol{w}=\left\{w_{c}\right\}$. Examples of the FCM clustering results are shown in Fig. 2.

\subsection{Acquisition of EMD flow}

The second step involves acquiring EMD flows from a source to a destination shape. Let sets of histogram bin means and weights for the source shape be $\overline{\boldsymbol{X}}^{s}, \boldsymbol{w}^{s}$ and those for the destination be $\overline{\boldsymbol{X}}^{d}, \boldsymbol{w}^{d}$, respectively. Then, the transportation cost and a flow (transportation amount) from the $j$ th bin of the source shape to the $k$ th bin of the destination shape are denoted as $t_{j k}$ and $f_{j k}$, respectively.

Though the transportation cost is typically defined as the Euclidean distance between the bin means $d_{j k}=\left\|\boldsymbol{x}_{j}^{s}-\boldsymbol{x}_{k}^{d}\right\|$, this sometimes induces an inhomogeneous work assignment, that is, a situation in which the transportation distances of a few flows are too long, while the majority of the others are relatively short. As 

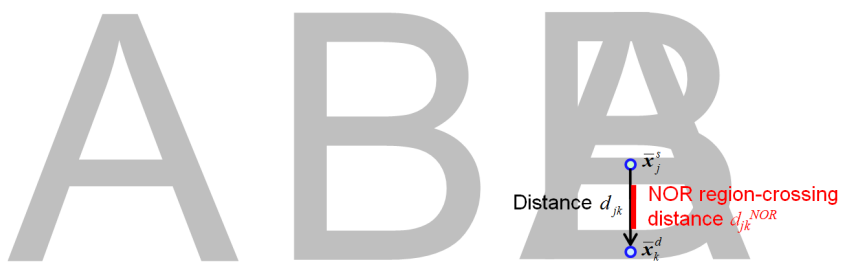

Fig. 3: NOR region-crossing distance.

this inhomogeneity is undesirable in morphing in particular, we use the squared Euclidean distance $t_{j k}=d_{j k}^{2}$ instead. Since the squared distance is more sensitive to a distant transportation, the transportation distances tend to be similar to one another, in other words, the clusters tend to move closer together.

Moreover, when a large deformation is necessary in the morphing process, it often happens that several flows cross a NOR (Not-OR) region as shown in Fig. 3. Although it depends on the particular situation whether or not crossing the NOR region is undesirable, this can be suppressed by adding a NOR regioncrossing distance to the transportation cost as

$$
t_{j k}=d_{j k}^{2}+\lambda^{N O R} d_{j k}^{N O R^{2}}
$$

where $\lambda^{N O R}$ is a coefficient of the NOR region-crossing distance.

Finally, the EMD flows are optimized in the following framework in conjunction with the Hungarian algorithm.

$$
\begin{aligned}
\left\{f_{j k}\right\}^{*}= & \arg \min _{\left\{f_{j k}\right\}} \sum_{j} \sum_{k} f_{j k} t_{j k} \\
\text { s.t. } & \sum_{k} f_{k l}=w_{k}^{s} \forall k \\
& \sum_{l} f_{k l}=w_{l}^{d} \forall l \\
& f_{k l} \geq 0 \forall k, l
\end{aligned}
$$

Now, we can regard the obtained $\left\{f_{j k}\right\}^{*}$ as the cluster-based many-to-many warping weight coefficients, whereas most of the existing methods use one-toone warping functions. Examples of the EMD flows and mean flows obtained from each cluster are shown in Fig. 4(a)(b).

\subsection{Cluster-based morphing}

The third step is the cluster-based morphing process using the obtained EMD flows $\left\{f_{j k}\right\}^{*}$. First, we consider a morphing from the $j$ th cluster in the source shape to the $k$ th cluster in the destination shape at a transition rate $\alpha$ as shown 


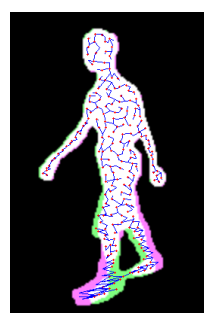

(a)

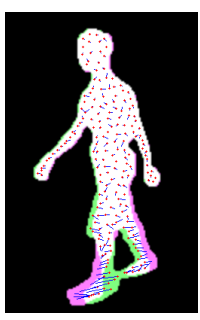

(b)

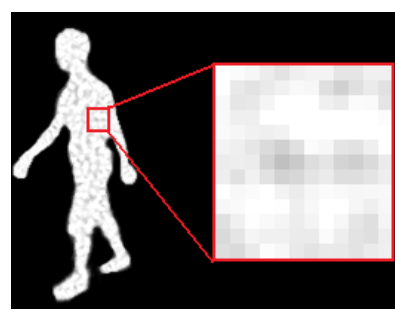

(c)

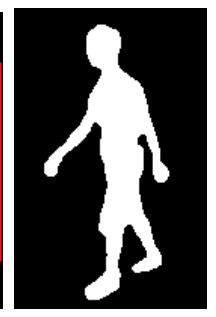

(d)

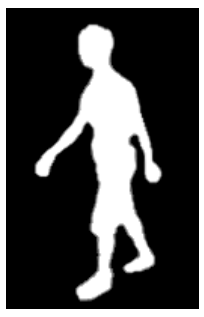

(e)

Fig. 4: EMD flows and morphing process between the key shapes in Fig. 2(a). (a) Raw EMD flows. (b) Mean flow of each cluster is calculated as a mean motion vector weighted by flow amount for visibility. (c) Blended morphing with artifacts. (d) Denoised binary morphing. (e) Boundary-dithered morphing.

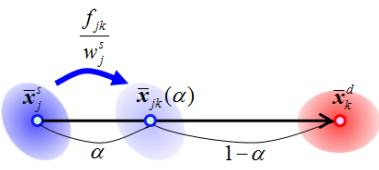

(a) Forward morphing

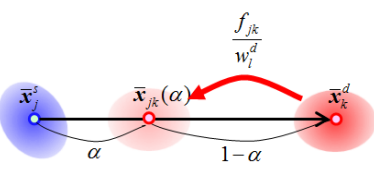

(b) Backward morphing

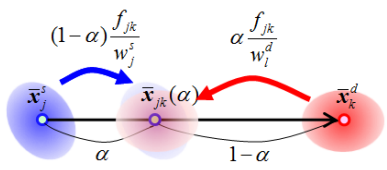

(c) Alpha blending

Fig. 5: Cluster-based morphing.

in Fig. 5. Next, let the interpolated position at the transition rate $\alpha$ between the $j$ th cluster mean $\overline{\boldsymbol{x}}_{j}^{s}$ and the $k$ th cluster mean $\overline{\boldsymbol{x}}_{k}^{d}$ be

$$
\overline{\boldsymbol{x}}_{j k}(\alpha)=(1-\alpha) \overline{\boldsymbol{x}}_{j}^{s}+\alpha \overline{\boldsymbol{x}}_{k}^{d}
$$

Then, suppose that a rate $\left(f_{j k} / w_{j}^{s}\right)$ of the $j$ th cluster is planned to be transported forward from $\boldsymbol{x}_{j}^{s}$ to $\boldsymbol{x}_{k}^{d}$ and that it is dropped at the interpolated point $\overline{\boldsymbol{x}}_{j k}(\alpha)$ on the way in the forward transportation as shown in Fig. 5(a). In this paper, we call this process forward morphs. The forward morphs for all the EMD flows are blended to create a forward morphing image $I_{\text {morph }}^{F}(\boldsymbol{x} ; \alpha)$. In the same way, a backward morphing image $I_{\text {morph }}^{B}(\boldsymbol{x} ; \alpha)$ is created (Fig. 5(b)) and then the forward morphing image $I_{\text {morph }}^{F}(\boldsymbol{x} ; \alpha)$ and backward morphing image $I_{\text {morph }}^{B}(\boldsymbol{x} ; \alpha)$ are alpha-blended to create a blended morphing image (Fig. 5(c)) as

$$
I_{\text {blend }}(\boldsymbol{x} ; \alpha)=(1-\alpha) I_{\text {morph }}^{F}(\boldsymbol{x} ; \alpha)+\alpha I_{\text {morph }}^{B}(\boldsymbol{x} ; \alpha)
$$

\subsection{Graph-cut denoising}

The last step is the denoising process for the blended morphing image $I_{\text {blend }}(\boldsymbol{x} ; \alpha)$. Unfortunately, the blended morphing image obtained by the cluster-based method suffers from "artifacts", that is, non-uniform silhouette intensity as shown in Fig. 4(c). Therefore, graph-cut denoising is applied to a volumetric blended morph image $I_{\text {blend }}(\boldsymbol{x} ; \alpha)$ with $2 \mathrm{D}$ spatial positions and a $1 \mathrm{D}$ transition parameter $\alpha$ to 
create a volumetric binary image $I_{b i n}(\boldsymbol{x} ; \alpha)$. Let a three-dimensional site and its label be $\boldsymbol{u}=\left[\boldsymbol{x}^{T}, \alpha\right]^{T}$ and $l_{\boldsymbol{u}}$, respectively. In this paper, the label is set to 1 for the silhouette region and to 0 otherwise.

Now, graph-cut denoising is formulated as the following energy minimization problem.

$$
E(L)=\sum_{\boldsymbol{u} \in U} g_{\boldsymbol{u}}\left(l_{\boldsymbol{u}}\right)+\sum_{(\boldsymbol{u}, \boldsymbol{v}) \in V} h_{\boldsymbol{u v}}\left(l_{\boldsymbol{u}}, l_{\boldsymbol{v}}\right),
$$

where $L$ is a combination of labels for each site, $U$ is the set of all sites, and $V$ is all the combinations of neighborhood sites. The first term is referred to as the data term and the second term as the smoothness term. The data term is determined based on the pixel intensity of the blended morphing image as

$$
g_{\boldsymbol{v}}(l)= \begin{cases}1-I_{\text {blend }}(\boldsymbol{x} ; \alpha) & (l=0) \\ I_{\text {blend }}(\boldsymbol{x} ; \alpha) & (l=1)\end{cases}
$$

The smoothness term is formulated by the Potts model as

$$
h_{\boldsymbol{u v}}\left(l_{\boldsymbol{u}}, l_{\boldsymbol{v}}\right)=\lambda_{\text {potts }}\left(1-\delta_{l_{\boldsymbol{u}} l_{\boldsymbol{v}}}\right),
$$

where $\lambda_{\text {potts }}$ is the smoothness term weight and $\delta$ is Kroneckerfs delta. Based on the data and smoothness terms defined above, the max-flow algorithm gives the globally optimized binary volumetric image $I_{b i n}(\boldsymbol{x} ; \alpha)$ (Fig. 4(d)).

Moreover, considering the effect of boundary dithering, boundary pixels are replaced by the blended morphing image to create the final resultant image $I_{\text {morph }}(\boldsymbol{x} ; \alpha)($ Fig. $4(\mathrm{e}))$ as

$$
I_{\text {morph }}(\boldsymbol{x} ; \alpha)= \begin{cases}I_{\text {blend }}(\boldsymbol{x} ; \alpha) & \boldsymbol{x} \text { is inner or outer boundary } \\ I_{b i n}(\boldsymbol{x} ; \alpha) & \text { otherwise. }\end{cases}
$$

As mentioned in 3.1, FCM clusters are preferable to k-means clustering in terms of denoising. They also tend to create a smoother blended morphing image than k-means clusters, with the result that artifacts such as holes and cracks in the silhouette region become less prominent, and are more easily recovered by graph-cut denoising.

\section{Experiments}

\subsection{Simple shapes}

In these experiments, several morphing examples of shapes with different genera are shown. The first example is the simplest, that is, morphing from a disk to an annulus as shown in Fig. 6 .

Starting from the disk $(\alpha=0.0)$, a silhouette hole appears near the center of the disk at $\alpha=0.2$ and the genus of the shape changes from 0 to 1 at this time. Then, the hole is gradually dilated as the transition parameter increases and the shape coincides with the annulus at the end of the transition $(\alpha=1.0)$. This kind of topological change is unique to the proposed approach. 


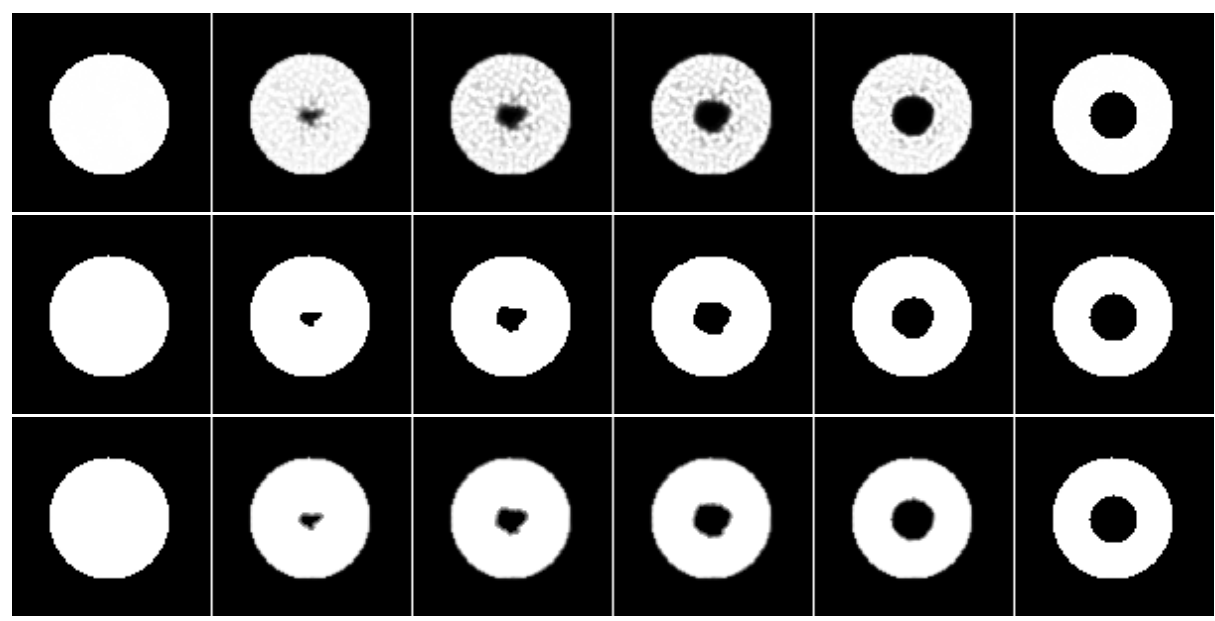

Fig. 6: Morphing from a disk (leftmost) to an annulus (rightmost). The middle four images are morphing images with transition parameters $\alpha=0.2,0.4,0.6$, and 0.8 , respectively. Top row: blended morphing, middle row: denoised binary morphing, bottom row: boundary-dithered morphing.

Although the artifacts are visible in the blended morphing (top row of Fig. 6), they are deleted by the graph-cut denoising (middle row of Fig. 6) and the boundary dithering (bottom row of Fig. 6) provides a visually desirable result.

Additional examples of morphing from a disk to double, triple, and quad annuli are shown in Fig. 7. In a similar manner to the previous example, multiple silhouette holes appear in the early stage of the transition and these are gradually dilated. Note that the number of holes appearing coincides with the genus of the corresponding destination shape.

\subsection{Real shapes}

The following examples involve gait silhouette morphs between two postures selected from gait silhouette sequences captured at a $60 \mathrm{fps}$ frame-rate. The source posture includes a silhouette hole in the leg region (leftmost image in Fig. 8) while the destination shape is expressed as a single closed curve (rightmost image in Fig. 8).

We can see that the resulting morphed images (the first and third rows of Fig. 8) are similar to the original gait sequences between the two postures (the second and fourth rows of Fig. 8). Therefore, the proposed method has real potential for use in many pattern recognition and image processing areas. For example, in a shape matching problem, intermediate shapes of the two key shapes can be generated for the purpose of training sample enhancements even in the presence of topological changes. In addition, when a low frame-rate sequence is provided in an action recognition or gait recognition problem, inter-frame silhouettes can 


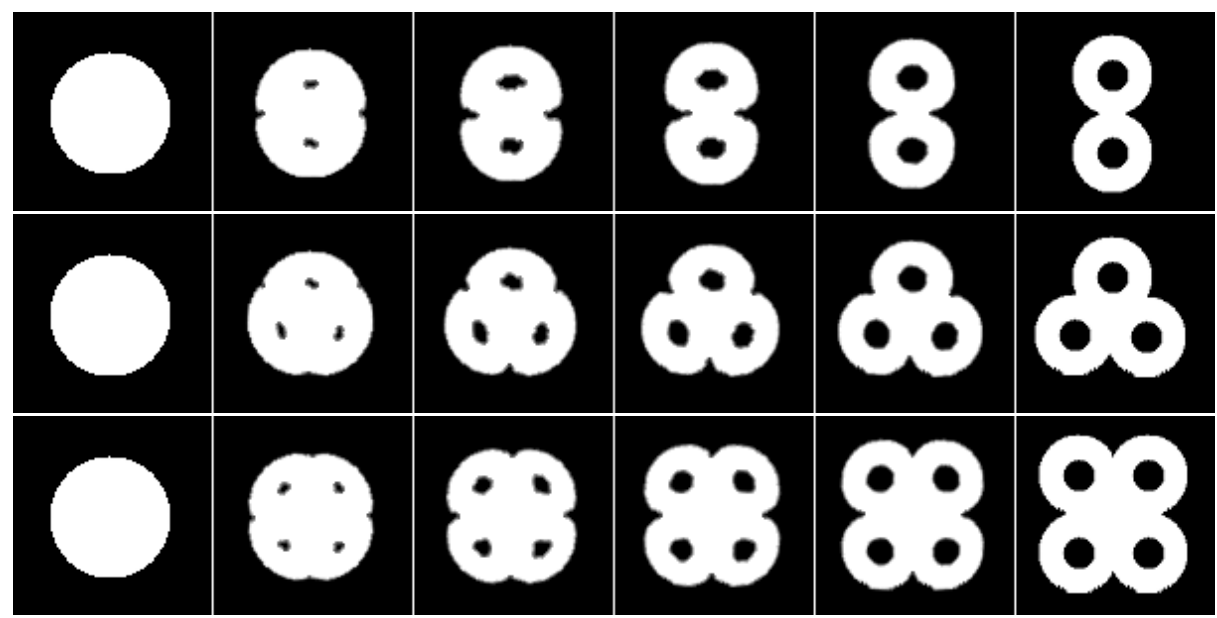

Fig. 7: Morphing from a disk (leftmost) to multiple annuli (rightmost). The middle four images show morphing with transition parameters $\alpha=0.2,0.4,0.6$, and 0.8 , respectively. Top row: double annulus, middle row: triple annulus, bottom row: quad annulus.

be interpolated and a temporal super-resolution sequence provided for better recognition without worrying about topological changes in the postures.

\subsection{Shapes with large deformation}

The third example shows morphing between alphabetical characters, which involves much larger deformation than in the previous two examples. First, we focus on morphing from "A" to "B". When the squared Euclidean distance $d_{j k}$ is used as the transportation cost $t_{j k}$, cluster flows around the horizontal middle bar in "A" are directed mainly in two directions: those that go upwards and others that go downwards across a wide NOR region as shown in the first row of Fig. 9. On the other hand, when the NOR region-crossing distance $d_{j k}^{N O R}$ is combined with the transportation cost $t_{j k}$, all the clusters in the middle bar go upwards across a narrower NOR region than the one in the row below (see the leftmost image in the second row of Fig. 9), as the flows crossing the wide NOR region below are penalized by additional transportation costs. As a result, isolated morphs that appear when using only the squared Euclidean distance are suppressed (see the second row of images in Fig. 9). The morphing from "B" to "C" is also a similar case with that from "A" to "B". While cluster flows of the middle horizontal bar of $\mathrm{B}$ go in various directions when using the squared Euclidean distance (the third row of images in Fig. 9), they are limited to three directions when the NOR region-crossing distance is added (the fourth row of images in Fig. 9).

This kind of character morphing can possibly serve as a novel transition effect technique for video editing applications. Compared with existing transitions 

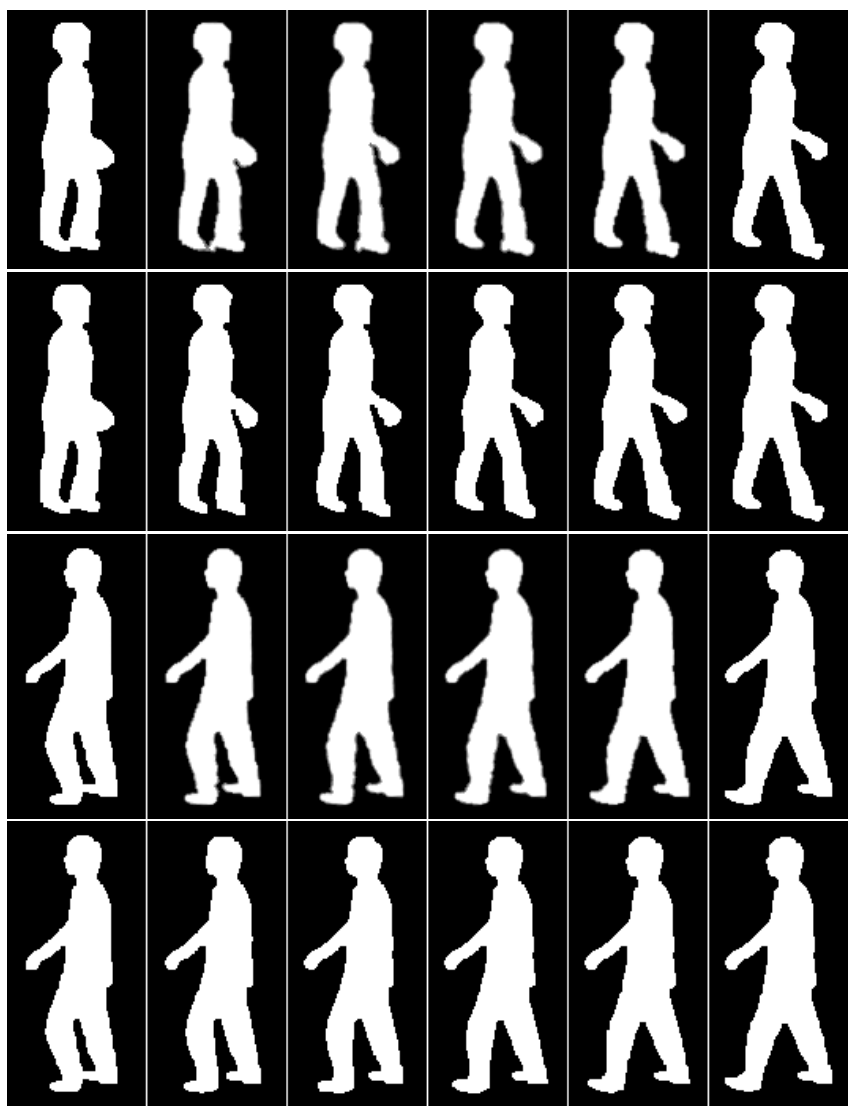

Fig. 8: Morphing from a 1 genus gait silhouette (leftmost) to a 0 genus one (rightmost). First and second rows: boundary-dithered morphing and an original gait sequence, respectively, from an oblique view. Third and fourth rows: the same, but from a side view.

such as cut, fade-in/out, slide, and wipe, the proposed morphing method provides a unique transition effect. In addition, considering the recent progress in interactive/automatic segmentation techniques [36], not only the characters in a title or caption scene, but also arbitrary objects' silhouettes, can be morphed once they have been extracted by a particular segmentation method. When applying morphing to such textured objects, color transfer on the morph should also be considered in the future.

\subsection{Split disks}

Our final example is morphing from a single disk to two split disks as shown in Fig. 10. If the signed distance field interpolation [23] is applied to this example, 


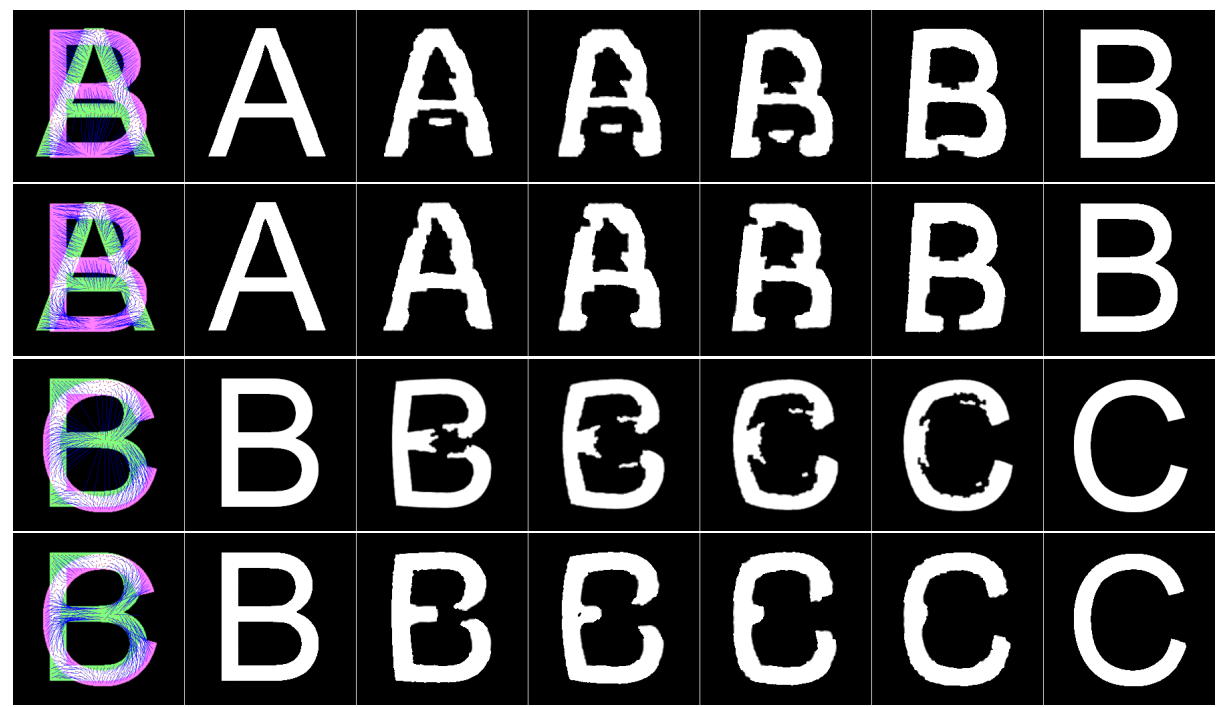

Fig. 9: EMD flows (leftmost) and morphing between alphabetical characters "A" (1 genus), "B" (2 genus) and "C" (0 genus). In EMD flow acquisition process, the squared Euclidean distance is used in each odd-row image, whereas the NOR region-crossing distance is used in conjunction with the squared Euclidean distance in each even-row image.

the source disk is eroded and finally disappears in the transition process, while the two destination disks appear as points in the centers and are dilated to the destination disks. By applying level set-based morphing [25], only the source disk is eroded and finally disappears in the transition process. Unlike these approaches, the proposed method gives a more interesting morphing process where the source disk is initially split into two hemisphere-like shapes, which then move to the destination position by changing their shapes from hemispheres to disks. This kind of morphing process is unique to the proposed method.

\section{Discussion}

In the proposed morphing process, a many-to-many correspondence of clusterbased EMD flows is used directly for cluster-based morphing. The automatically obtained correspondence is also provided to construct the existing warping functions [7] [8] [9] [10] [11] [12] between shapes with arbitrary genera.

Another point is that the cluster-based EMD flow can be applied to achieve many purposes, that is, not only morphing, but also shape matching, deformable model construction, and motion analysis without worrying about topological changes, since existing contour-based methods [31] are used within the closed curve/surface shapes. Unlike the optical flows extracted from a textured image sequence that correspond to real motion, the region cluster-based EMD flows 


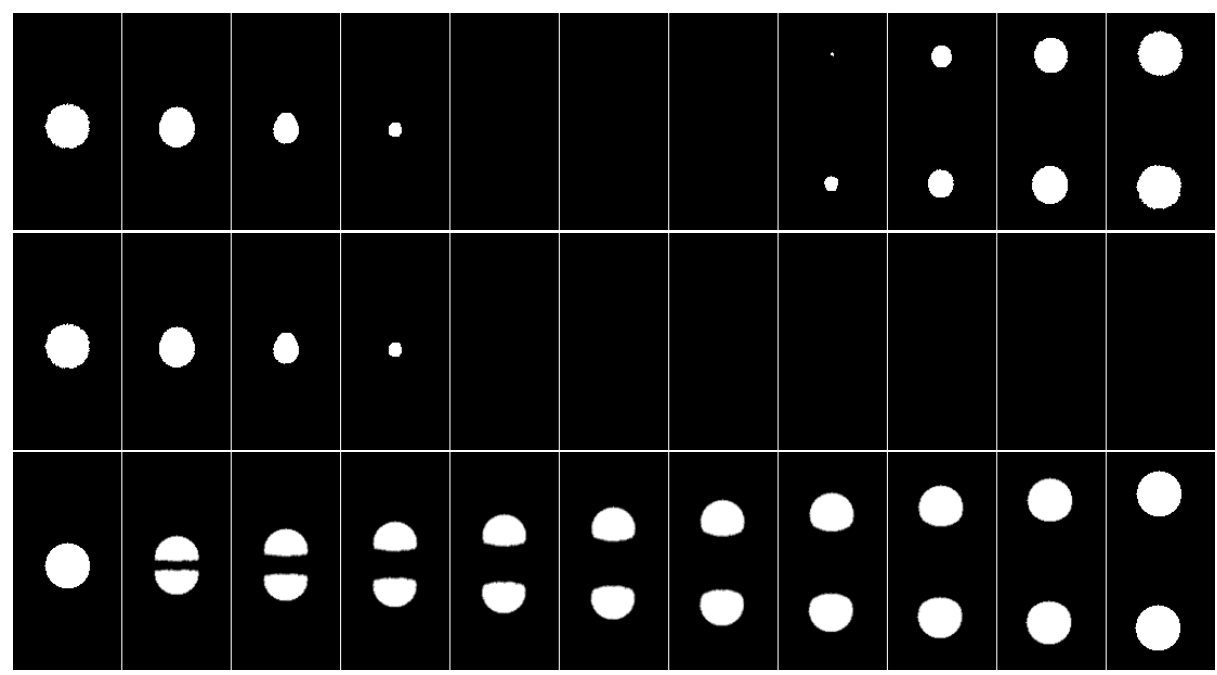

Fig. 10: Morphing from a single disk (leftmost) to two split disks (rightmost). Top row: signed distance field interpolation [23], middle row: narrow-band level-set method [25], bottom row: the proposed method.

do not correspond to real motion, but to pseudo motion. This pseudo motion, however, still has potential as a novel motion feature for silhouette-based motion analysis.

On the other hand, the EMD framework allows too much flex flows in several cases (e.g., large deformation of alphabetical characters in 4.3). We need to introduce additional schemes such as regularity constraints and non-linear interpolation in order to maintain shape well during the morph in the future.

\section{Conclusion}

This paper described a method for topology-free shape morphing based on region cluster-based EMD flows. First, the region was decomposed into a number of small clusters by FCM clustering and a histogram of the clusters was constructed. Next, the EMD between the two histograms was calculated with the resultant flows and position displacement between the clusters serving as a weighted many-to-many correspondence. A fuzzy cluster-based morphing transition was provided by the obtained correspondence. Finally, the three-dimensional graph-cut binary denoising was applied to reduce artifacts caused by the clusterbased morphing.

Future works are listed below.

- Texture transfer on the morph in conjunction with automatic/interactive segmentation.

- Warping function reconstruction from the weighted many-to-many correspondence based on the EMD flows. 
- Application of pseudo motion within the shape to silhouette-based recognition, as in action recognition and gait-based person identification.

Acknowledgement. This work was supported by Grant-in-Aid for Scientific Research(S) 21220003.

\section{References}

1. Wolberg, G.: Image morphing: a survey. The Visual Computer 14 (1998) 360-372

2. Seitz, S.M., Dyer, C.R.: View morphing. In: Proc. of ACM SIGGRAPH1996. (1996) 21-30

3. Blanz, V., Vetter, T.: A morphable model for the synthesis of $3 \mathrm{~d}$ faces. In: In Proc. of ACM SIGGRAPH. (1999)

4. Beymer, D., Poggio, T.: Image representations for visual learning. Science 272 (1995) 1905-1909

5. Cootes, T., Taylor, C., Cooper, D., Graham, J.: Active shape models - their training and application. Computer Vision and Image Understanding 61 (1995) 38-59

6. Cootes, T., Edwards, G., Taylor, C.: Active appearance models. IEEE TPAMI 23 (2001) 681-685

7. Wolberg, G.: Digital image warping. IEEE Computer Society Press (1990)

8. Beier, T., Neely, S.: Feature-based image metamorphosis. Computer Graphics 26 (1992) 35-42

9. Arad, N., Dyn, N., Reisfeld, D., Yeshurun, Y.: Image warping by radial basis functions: applications to facial expressions. CVGIP: Graph Models Image Processing 56 (1994) 161-172

10. Lee, S., Chwa, K.Y., Hahn, J., Shin, S.: Image morphing using deformable surfaces. In: Procc of Computer Animation'94, IEEE Computer Society Press (1994) 31-39

11. Chwa, K.Y., Hahn, J., Shin, S.: Image morphing using deformation techniques. J. of Visualization Computer Animation 7 (1996) 3-23

12. Lee, S.Y., Chwa, K.Y., Shin, S.Y.: Image metamorphosis using snakes and free-form deformations. In: SIGGRAPH '95: Proceedings of the 22nd annual conference on Computer graphics and interactive techniques, New York, NY, USA, ACM (1995) 439-448

13. Lee, S., Wolberg, G., yong Chwa, K., Shin, S.Y.: Image metamorphosis with scattered feature constraints. IEEE Transactions on Visualization and Computer Graphics 2 (1996) 337-354

14. Gao, P., Sederberg, T.W.: A work minimization approach to image morphing. The Visual Computer 14 (1998) 390-400

15. Toshev, A., Shi, J., Daniilidis, K.: Image matching via saliency region correspondences. In: Proc. of IEEE computer society conferene on Computer Vision and Pattern Recognition. (2007) 1-8

16. Tekalp, M.: Digital video processing. Prentice Hall PTR (1995)

17. Chen, S.E., Williams, L.: View interpolation for image synthesis. In: Proc. of ACM SIGGRAPH1993. (1993) 279-288

18. Shechtman, E., Rav-Acha, A., Irani, M., Seitz, S.M.: Regenerative morphing. In: Proc. of of IEEE computer society conferene on Computer Vision and Pattern Recognition 2010, San Francisco, CA, USA (2010) 1-8

19. Sederberg, T.W.: A physically based approach to 2-d shape blending. Computer Graphics 26 (1992) 
20. Kent, J., Carlson, W., Parent, R.: Shape transformation for polyhedral objects. Computer Graphics 26 (1992) 47-54

21. DeCarlo, D., Gallier, J.: Topological evolution of surfaces. In: Proc. Graphics Interface. (1996) 194-203

22. Fu, H., Tai, C.L., Zhang, H.: Topology-free cut-and-paste editing over meshes. In: GMP '04: Proceedings of the Geometric Modeling and Processing 2004, Washington, DC, USA, IEEE Computer Society (2004) 173

23. Payne, B., Toga, A.: Distance field manipulation of surface models. IEEE Computer Graphics and Applications 12 (1992) 65-71

24. Cohen-Or, D., Levin, D., Solomivici, A.: Three-dimensional distance field metamorphosis. ACM Trans. Graphics 17 (1998) 116-141

25. Breen, D., Whitaker, R.: A level-set approach for the metamorphosis of solid models. IEEE Transactions on Visualization and Computer Graphics 7 (2001) $172-192$

26. Castro, G., Ugail, H.: Shape morphing of complex geometries using partial differential equations. Journal of Multimedia 2 (2007) 15-25

27. Osher, S., Sethian, J.: Fronts propagating with curvature dependent speed: Algorithm based on hamilton-jacobi formation. Journal of Computational Physics $\mathbf{7 9}$ (1988) 12-49

28. Zhu, L., Yang, Y., Tannenbaum, A., Haker, S.: Image morphing based on mutual information and optimal mass transport. In: Int. Conf. on Image Processing 2004. (2004) 1675-1678

29. Zhu, L., Yang, Y., Haker, S., Tannenbaum, A.: An image morphing technique based on optimal mass preserving mapping. IEEE Transactions on Image Processing 16 (2007) 1481-1495

30. Kaziska, D., Srivastava, A.: Cyclostationary processes on shape spaces for gaitbased recognition. In: In proc. of European Conf. on Computer Vision. 2 (2006) 442-453

31. Grauman, K., Darrell, T.: Fast contour matching using approximate earth mover's distance. In: IEEE Computer Society Conf. on Computer Vision and Pattern Recognition. (2004) 220-227

32. Bronstein, A.M., Bronstein, M.M., Kimmel, R.: Numerical Geometry of Non-Rigid Shapes. Springer (2008)

33. Schneider, D.C., Eisert, P.: Fast nonrigid mesh registration with a data-driven deformation prior. In: In Proc. ICCV Workshop on Non-Rigid Shape Analysis and Deformable Image Alignment (NORDIA). (2009)

34. Sagawa, R., Akasaka, K., Yagi, Y., Hamer, H., Gool, L.V.: Elastic convolved icp for the registration of deformable objects. In: In Proc. 2009 IEEE 12th International Conference on Computer Vision Workshops (3DIM2009), Kyoto, Japan (2009) $1558-1565$

35. Hoppner, F., Klawonn, F., Kruse, R., Runkler, T.: Fuzzy Cluster Analysis. John Wiley and Sons (1999)

36. Li, Y., Sun, J., Shum, H.Y.: Video object cut and paste. ACM Trans. Graph. 24 (2005) 595-600 